\title{
Gründungsberatung im Spannungsfeld zwischen Innovation und Kreativität
}

\author{
Ullrich Beumer • Heidi Möller
}

Online publiziert: 21. Januar 2019

(C) Springer Fachmedien Wiesbaden GmbH, ein Teil von Springer Nature 2019

Innovationsfähigkeit gilt zunehmend als Schlüsselkompetenz für erfolgreiche Unternehmensentwicklung. Das Schlagwort „Innovation“ beginnt, im Unternehmensbereich dem „Change“ als Dauerpostulat den Rang abzulaufen. Parallel entstehen zahlreiche methodische Konzepte, die der Förderung von Innovation dienen, hier insbesondere das „Design Thinking“, das in rasanter Geschwindigkeit zunehmend in den Portfolios von Beratern und Beratungsunternehmen auftaucht.

Die institutionelle Entsprechung bzw. Weiterentwicklung der Innovation ist die Existenzgründung. In vielerlei Formen versuchen insbesondere jüngere Menschen, ihrem Drang nach Neuerung durch die Gründung von Start-Ups, kleinen, agilen Firmen und Projekten Ausdruck zu verleihen. „Wecke den Gründer in Dir“ - so lautet die Maxime auf dem Gründerportal des Bundesministeriums für Wirtschaft und Energie. Gründer zu sein dient dabei idealerweise sowohl der Gesamtwirtschaft und der gesellschaftlichen Entwicklung als auch der eigenen Entfaltung der Persönlichkeit des Gründers. Neben der Geschäftsidee und dem notwendigen Kapital ist die Person des Gründers dabei die dritte zentrale Variable für einen erfolgreichen Gründungsprozess.

Gründercoaching gehört seit langem neben der Einrichtung von Gründerzentren zu den unterstützenden Maßnahmen, die Geldgeber und öffentliche Institutionen angehenden Existenzgründern anbieten, um den Neuaufbau zu begleiten und zu sichern. Wie aber arbeiten Gründungsberater? Existenzgründungen sind nicht nur eine Frage von Produktideen, Businessplänen, Marktanalysen und gezielter Nutzung

Dr. U. Beumer $(\bowtie)$

inscape, Riehler Straße 23, 50668 Köln, Deutschland

E-Mail: Ullrich.Beumer@inscape-international.de

Prof. Dr. H. Möller

Institut für Psychologie, Universität Kassel, Holländische Straße 36-38, 34127 Kassel, Deutschland

E-Mail: heidi.moeller@uni-kassel.de 
von Fördermöglichkeiten. Sie bedeuten eine gravierende Bahnung der beruflichen Entwicklung der jeweiligen Person und haben Auswirkungen auf die persönliche Situation und das soziale Umfeld. Die Qualität der Gründungsberatung wächst mit der Fähigkeit, Chancen und Herausforderungen für die Existenzgründer/innen wahrzunehmen und ihnen angemessene Hilfestellung an die Hand zu geben. In welcher Form sind sie kompetent und bereit, sich den oft komplexen Fragen der Persönlichkeit zu stellen? Darüber hinaus lässt sich die Frage stellen, ob Professionen wie z. B. die Supervision, die über wertvolle Kompetenzen in der Beratung auf der personalen Ebene verfügen, auf Gründer eingestellt sind und diese als relevante Zielgruppe ihrer Beratung im Blick haben.

Diesen Fragen im Spannungsfeld von Kreativität, Innovation und Existenzgründung widmen sich die Beiträge des Hauptteils dieses Hefts. Es handelt sich dabei größtenteils um überarbeitete Vorträge, die auf dem 8. Kongress für psychodynamisches Coaching, 23.-24.02.2018, Universität Kassel, gehalten worden sind.

Ullrich Beumer stellt als Ergebnis seiner empirischen Untersuchung mit älteren ehemaligen Führungskräften die besondere Situation und Motivation älterer männlicher Existenzgründer vor, die ein Beratungsunternehmen gegründet haben. Zwar sind laut einer Umfrage $78 \%$ der 50- bis 75-Jährigen für einen (beruflichen oder persönlichen) Neustart offen, aber nur ein verschwindend kleiner Prozentsatz von ihnen entscheidet sich tatsächlich, ein eigenes Unternehmen zu gründen bzw. eine Freiberuflichkeit als „Solopreneur“ zu starten. Der Autor beschreibt, wie diese Existenzgründungen weniger von der Lust am Risiko geprägt sind, sondern von einem reflektierten Umgang mit der eigenen Karriere, Lebensgeschichte und von dezidierten Vorstellungen zur beruflichen und persönlichen Zukunft jenseits des Renteneintritts. Existenzgründungen in der zweiten Lebenshälfte „heilen“ Beschädigungen, die Führungskarrieren mit sich bringen, und schaffen Raum für unerfüllte Wünsche und Lebenspläne. Auf der Basis von biografischen Interviews mit ehemaligen Führungskräften, die sich jenseits der 50 selbstständig gemacht haben, wird eine Typologie der späten Existenzgründung entwickelt.

Unternehmensgründungen werden noch immer vorwiegend von Männern getätigt. In Kontrast zu dieser empirischen Realität werden Frauen ebenso wie ältere Existenzgründer politisch als eine Zielgruppe mit ungenutztem Potenzial angesehen. Annett Adler und Brigitte Halbfas zeigen in ihrem Beitrag auf, wie sich in der Gründungsberatung Praktiken des Doing Gender darstellen. Sie verknüpfen dabei die drei analytischen Ebenen von politischer und wirtschaftlicher Situiertheit, der wissenschaftlichen Situiertheit und die Situation der Gründungsberatung als empirisches Beispiel selbst. Ausgehend von den Ergebnissen eines Forschungsprojekts werden Forderungen für Organisationsberatung, Coaching und Supervision als Teil der Gründungsberatung entwickelt.

Durch zahlreiche wissenschaftliche Untersuchungen ist inzwischen nachgewiesen, dass das Gelingen von Existenzgründungen Ergebnis eines komplexen Zusammenspiels von Gründerpersönlichkeit und Gründungsmotivation, einer entsprechenden Gründungsidee und förderlichen situativen Rahmenbedingungen ist. Gleichwohl kommt es in der Gründungsberatung darauf an, die Person des Gründers angemessen zu beraten und bei ihrer Entscheidung zu überprüfen, ob sie über ausreichende Kompetenzen verfügent, den Prozess auch erfolgreich umzusetzen. Martin Seip be- 
schreibt den Aufbau des „Gründerkompasses“, eines Assessmentcenters zur kompetenzorientierten Diagnostik gründungsrelevanter Fähigkeiten als wichtigem Beitrag zu einer wirksamen Gründungsberatung. Als Perspektive wird ein systematisches Coaching auf der Grundlage der im Assessmentcenter gewonnenen Daten vorgeschlagen.

Zum guten Ton in Unternehmen gehört es heute, ,innovativ zu sein“, und viel Energie und Geld wird auf die Implementierung entsprechender Methoden und Strukturen verwandt. Viele dieser Konzepte sind jedoch rein äußerlich, sie zeigen sich eher als eine Art „Innovation der Buchhalter“. Echte Innovationen gelingen dagegen nur, wenn ein Zugang zur Kreativität im gesamten Unternehmen gefördert wird. Silke Facilides plädiert für eine grundlegende Kulturveränderung in Unternehmen, für mehr kreatives Denken, um in einer komplexer werdenden Welt dauerhaft erfolgreich - und innovativ - zu bleiben. Die von ihr vorgestellten Konzepte basieren auf Erfahrungen aus der „Kreativindustrie“, verbunden mit dem persönlichen Umgang mit Kreativität und dem Glauben an Kreativität als eine urmenschliche Fähigkeit. Unternehmen, die dauerhaft innovativ sein wollen, sollten sich auf eine ständige kreative Sinnsuche einlassen und emotionale Resonanz als Treiber von Kreativität willkommen heißen, statt den Innovationsdruck zu erhöhen.

Innovation als prozesshafte Veränderung innerhalb von Organisationen ist eine Voraussetzung für Gründungsimpulse und die Entscheidung, gesicherte Verhältnisse zu verlassen, um sich als Entrepreneur zu riskieren. Das Verhältnis der Gesellschaft zu (wirtschaftlichen bzw. technischen) Neuerungen bzw. Innovationen hat sich innerhalb der letzten 100 Jahre in modernen Gesellschaften von Ablehnung hin zur Alltäglichkeit gewandelt. Birgit Blättel-Mink gibt einen Überblick über das Innovationskonzept in Wirtschaft und Gesellschaft und die Innovationsbemühungen der vergangenen Jahrzehnte. In ihrem Beitrag wird, ausgehend vom ,reinen“ Unternehmer bei Joseph A. Schumpeter, ein kurzer Abriss der zentralen Ansätze der Innovationsforschung gegeben. Neben der Institutionalisierung von Innovation geht es in einem zweiten Diskursstrang um den Wandel von einer individualistischen Perspektive auf den/die Träger/in der Innovation hin zu einer Netzwerk- bzw. Systemperspektive.

Im Praxisbericht dieses Heftes geht Pierre-Pascal Urbon der Frage nach, was den Erfolg von Start-ups ausmacht. Dazu stellt er einen Vergleich mit erfolgreichen Start-ups im Silicon Valley an, weil es dort eine ganz andere Gründerkultur als in Deutschland zu geben scheint, und er führt aus, was wir in Deutschland davon lernen können. Dies wird am Beispiel von SMA Solar Technology AG in Niestetal erläutert, für die der Autor 2006-2018 als Vorstandssprecher tätig war.

Neben diesen Beiträgen zum Themenschwerpunkt bietet das Heft einen weiteren Hauptbeitrag: Silja Kotte, Elisabeth Bick, Cord Benecke und Heidi Möller präsentieren die Operationalisierte Psychodynamische Diagnostik (OPD) im Arbeitskontext und stellen dazu die Entwicklung eines berufsbezogenen Konflikt-Fragebogens vor. Zunächst wird die Spezifik der psychodynamischen Diagnostik für die Personalauswahl einerseits, andererseits für die Eingangs-, Verlaufs- und Abschlussdiagnostik im Coaching herausgearbeitet. Der psychodynamische Blick bietet hier zusätzliche Perspektiven, die die traditionellen Diagnosemöglichkeiten übertreffen. Das in der Entwicklung befindliche Instrument wird Personalern, Coaches, Supervisoren und 
Organisationsberatern eine hilfreiche wissenschaftliche Fundierung für präventive Maßnahmen als auch für ihr intervenierendes Handeln sein.

In unserem Diskurs wird das Dauerbrennerthema „Coaching durch die Führungskraft" von Wolfgang Kühl, Erich Schäfer und Andreas Lampert vor dem Hintergrund agiler Arbeitswelten und der sich neu definierenden Führungsrolle anders eröffnet und mit zahlreichen Argumenten unterlegt. Lippmann und Schreyögg antworten mit ihren unterschiedlichen Perspektiven, die jedoch einen gemeinsamen Nenner haben: das Plädoyer für eine saubere konzeptionelle Trennung der Entwicklungsunterstützung durch die Führungskraft versus der externen Coachingformate.

In der Rubrik Kulturanalysen stellt Thomas Giernalczyk die Organisationskultur eines Familienunternehmens am Beispiel des Films Väter und Söhne von Bernhard Sinkel vor. „Wer die Formel kennt, hat die Macht“ zeigt, wie vielschichtig der Wunsch nach Generativität durch zahlreiche - oftmals unbewusste - nicht nur ödipale Generationskonflikte unterlegt ist. Familie und Unternehmen stellen immer ein brisantes Spannungsfeld zwischen Liebe und Geld dar. Familiendynamik, Eigentum und Unternehmen interagieren als gekoppelte Systeme mit verwobenen, intensiven und ambivalenten Auswirkungen des Unternehmens auf die Familienstruktur und die persönlichen Biografien der Protagonisten.

Ullrich Beumer und Heidi Möller wünschen Freude beim Erkenntnisgewinn! 